\title{
A Study of Bio-Markers of Oxidative Stress and Inflammation in Chronic Kidney Disease
}

\author{
${ }^{1}$ Meenakshi Sreeram, ${ }^{2}$ Suryakar AN, ${ }^{3}$ Kulhalli PM \\ ${ }^{1}$ Dept of Biochemistry, MGV's Dental College \& Hosp, Nasik/ MUHS, Nasik/ Maharashtra, India. \\ ${ }^{2}$ Registrar, Maharashtra University of Health Sciences (MUHS), Nasik/ Maharashtra, India. \\ ${ }^{3}$ University Department of Interpathy Research (UDIRT)/MUHS, Nasik/Maharashtra, India.
}

\begin{abstract}
Chronic Kidney Disease (CKD) is one of the most important health challenges faced by the world today, with new cases being added at an alarming rate. Controlling traditional risk factors has not been effective in bringing down the incidence of the disease. CKD is associated with inflammation, morbidity, poor quality of life, decreased life-expectancy and death. Oxidative stress represents the imbalance between prooxidants and antioxidants in our body. Though a number of studies have focussed on the association between $C K D$ and oxidative stress, majority of them are based on patients on hemodialysis. Moreover, there is confusion about the way the levels of some of these biomarkers vary in response to oxidative stress. This study was designed to study the biomarker of inflammation (CRP), MDA as a marker of oxidative stress, and SOD, GPx and TAC as parameters of antioxidant activity in controls and patients in different stages of CKD. We found that CRP and MDA values increase as disease progresses while there is decrease in the levels of SOD and GPX. The TAC values in patients do not vary much from that of controls. Efforts should be made to identify and reduce oxidative stress from the time of diagnosis of CKD.
\end{abstract}

KEY WORDS: Chronic Kidney Disease, Glutathione peroxidase, Inflammation, Malondialdehyde, Oxidative Stress, Superoxide dismutase, Total Antioxidant Capacity

\section{INTRODUCTION}

Chronic Kidney Disease (CKD) also known as chronic renal disease, is a progressive loss in renal function over a period of months or years. CKD and its resultant sequelae represent a major challenge to the medical world. The prevalence is estimated to be $8-16 \%$ worldwide. ${ }^{[1]}$ While developed countries are allocating large funds to face this challenge, people in the developing countries are reeling under the tremendous financial, psychological and emotional burden of the disease due to insufficient infrastructure and resources available to deal with the disease. Scientists are looking at not just traditional risk factors but also non-traditional ones to help in early detection of the disease so as to initiate treatment early and probably try to reverse this condition or at least delay adverse outcomes.

Reactive Oxygen Species (ROS) are free radicals which are constantly being produced in our body during various metabolic processes. When healthy, our body is endowed with the ability to deal with these attackers with the help of antioxidant mechanisms. Trouble starts brewing when the subtle balance tilts in favour of the free radicals. They gain an upper hand while the defence mechanism lags behind. Cellular antioxidants are overwhelmed by repeated oxidative insults. The production of ROS soon cascades leading to a scenario of tissue damage, cell death and disease. ROS cause tissue damage by a variety of different mechanisms which include DNA \& protein damage, lipid peroxidation, stimulating release of pro-inflammatory cytokines, etc. ${ }^{[2]}$ This disturbance in the delicate equilibrium of pro-oxidants:antioxidants in favour of the pro-oxidants is termed as oxidative stress. In short, oxidative stress represents an imbalance between the production and manifestation of ROS and a biological system's ability to readily detoxify the reactive intermediates or to repair the resulting damage. Oxidative stress may be the sole cause of some disease but more often it weakens the immune system and makes the body vulnerable to diseases caused by other factors. It may also worsen existing conditions and slow down healing process.

CKD is associated with increased inflammation. ${ }^{[3]}$ Chronic inflammation in the body is measured by the elevated levels of inflammatory markers and CRP is the marker of choice in monitoring the acute phase response as it increases to a relatively high concentration compared to basal concentration. ${ }^{[4]}$ Many studies in the recent times have been harvesting increasing evidence about the association between oxidative stress and $\mathrm{CKD}$, and, many markers are being routinely used to measure this. ${ }^{[5-7]}$ There is still confusion about how the levels of some of these markers vary with increasing severity of the disease. Moreover, many of the studies in this regard are based on patients on hemodialysis (HD). So, we set out to measure markers of inflammation and parameters of oxidative stress in patients in different stages of CKD. 


\section{MATERIALS AND METHODS}

The present study was carried out in the Department of Biochemistry, MGV's KBH Dental College and Hospital, Nasik and Supreme Kidney Care, Om Chambers, Nasik.

The protocol for this cross-sectional study was approved by the Institutional Ethics Committee of MGV's KBH Dental College and Hospital, Nasik and the Human Ethics Committee at UDIRT, Maharashtra University of Health Sciences, Nasik. Prior to enrolment in the study, candidates who fulfilled the inclusion criteria were given detailed oral information about the study and invited to participate. A Patient Information Sheet (PIS) was handed over to them and written informed consent obtained.

108 patients of age $\geq 18$ years with pre-diagnosed CKD and 22 controls were included in this study. The subjects were enrolled from Supreme Kidney Care, Nasik.

\subsection{Inclusion Criteria}

\subsubsection{Controls}

These were the relatives/ friends accompanying the patients to the OPD. These volunteers had

- No previous diagnosis of CKD.

- No other systemic complications.

\subsubsection{Study group subjects}

Study group subjects were people with confirmed diagnosis of CKD of any cause for at least 6 months.

\subsection{Exclusion Criteria}

Both controls and study group subjects were excluded if they

- Had undergone anti-microbial/ anti-inflammatory therapy in the last 6 months.

- Were on vitamin supplements.

- Used steroidal or immunosuppressive drugs.

- Consume alcohol.

- Are pregnant/lactating women or women on hormone-replacement therapy.

- Have acute inflammatory illnesses, hospitalization for cardiac or infection-related morbidity within 6 weeks before study, severe comorbid complications, previous kidney transplantation or are on experimental drug protocols.

\subsection{Sample Collection}

Blood samples were obtained from the patients and controls in the mornings during regular outpatient departmental visit. A total of $5 \mathrm{ml}$ blood was collected from each subject, $2 \mathrm{ml}$ was collected in heparinised bulb and the remaining was collected in plain bulb. Plasma was separated by centrifugation at $3000 \mathrm{rpm}$ for 10-15 minutes at room temperature. Samples for serum collection were allowed to clot at room temperature before centrifugation. All samples were analyzed on the same day of collection.

\subsection{Biochemical Parameters}

The following tests were performed:

1. Estimation of serum C-reactive protein (CRP) by( Immunoturbidometric Method using Turbilyte CRP kit from Tulip Diagnostic (P) Ltd., Goa, India.

2. Estimation of serum lipid peroxide level by the method of Kei Satoh. ${ }^{[8]}$

3. Estimation of Superoxide dismutase (SOD) activity in serum by method of Kajari Das. ${ }^{[9]}$

4. Estimation of Glutathione Peroxidase (GPx) in serum by method of Paglia and Valentine. ${ }^{[10]}$

5. Estimation of Total Antioxidant Capacity (TAC) by FRAP method of Benzie et al. ${ }^{[11]}$

\section{RESULTS}

The comparison of control group was carried out with the study group with respect to mean value \pm standard deviation. For comparison of parameters between controls and various stages of CKD, one-way analysis of variance (ANOVA) was applied. The results obtained are tabulated below. Probability values of $<0.05$ were considered to be statistically significant.

Table 1: Demographics of controls and CKD patients

\begin{tabular}{|l|l|l|l|l|}
\hline Group & Number & Males & Females & $\begin{array}{c}\text { Mean Age } \\
\text { (Years) }\end{array}$ \\
\hline Controls & 22 & 13 & 09 & $42.86 \pm 10.59$ \\
\hline Stage 1+2 & 30 & 22 & 08 & $32.93 \pm 10.96$ \\
\hline Stage 3+4 & 40 & 28 & 12 & $49.98 \pm 10.94$ \\
\hline Stage 5 & 38 & 23 & 17 & $49.39 \pm 14.55$ \\
\hline
\end{tabular}


Table 2: Mean values of CRP \& Parameters of Oxidative Stress in controls and patients

\begin{tabular}{|l|l|l|l|l|l|}
\hline Parameter & Controls & Stage 1\& 2 & Stage 3 \&4 & Stage 5 & P value \\
\hline CRP $(\mathrm{mg} / \mathrm{dl})$ & $1.33 \pm 1.03$ & $2.15 \pm 0.99$ & $5.88 \pm 3.20$ & $5.54 \pm 3.74$ & $<0.001$ \\
\hline MDA (nmol/ml) & $5.26 \pm 0.64$ & $5.79 \pm 0.79$ & $7.53 \pm 0.59$ & $7.68 \pm 0.55$ & $<0.001$ \\
\hline SOD (U/L) & $4.34 \pm 0.72$ & $3.78 \pm 0.43$ & $3.45 \pm 0.52$ & $3.19 \pm 0.62$ & $<0.001$ \\
\hline GPx (U/ml) & $3.58 \pm 0.40$ & $3.40 \pm 0.30$ & $3.25 \pm 0.44$ & $2.97 \pm 0.39$ & $<0.001$ \\
\hline TAC (mmol/lit) & $1.97 \pm 0.13$ & $1.63 \pm 0.30$ & $1.94 \pm 0.16$ & $2.00 \pm 0.23$ & $<0.001$ \\
\hline
\end{tabular}

TABLE 1 represents the demographic characteristics of controls and cases. Mean age of controls in our study is more than that of patients with stage $1 \& 2$ CKD while stage $3 \& 4$ and stage 5 patients are almost in the same age group. There are more number of males as compared to females in all the groups. TABLE 2 depicts the mean values of all parameters of oxidative stress in controls and cases. As expected, mean CRP levels are found to be lowest in the controls, followed by stage $1 \& 2$. The mean CRP values in stage $3 \& 4$ and stage 5 CKD patients are found to be very close to each other. MDA values show a gradual increase from controls to stage 5 CKD, while SOD and GPx values show a decreasing trend. Stage $1 \& 2$ CKD patients have lower TAC values as compared to controls, while stage $3 \& 4$ and stage 5 CKD patients are found to have TAC values comparable to that of controls.

\section{Discussion}

Once the diagnosis of $\mathrm{CKD}$ is made, staging is based on the estimated glomerular filtration rate (eGFR). Recent professional guidelines classify the severity of CKD in 5 stages, with stage 1 being the mildest and usually causing few symptoms and stage 5 being a severe illness with poor life expectancy if untreated. ${ }^{[12]}$

Our results show that controls have lower mean CRP values as compared to the CKD patients. As the disease progresses, it results in more severe inflammation. Stage $3 \& 4$ and stage 5 CKD patients show the highest CRP values. Inflammation is associated with increased oxidative stress. ${ }^{[13]}$ This in turn results in increased lipid peroxidation leading to increase in the levels of Malondialdehyde (MDA). In our study, we found that MDA levels are lower in controls as compared to patients with CKD. As compared to early stages of $\mathrm{CKD}$, later stages of CKD exhibit significantly higher levels of MDA, which implies that oxidative stress increases with disease progression. SOD and GPx are the enzymes of antioxidant defence in our body. As oxidative stress increases, the defence mechanism is overwhelmed by the repeated onslaught of the ROS, resulting in lower levels of these enzymes. It is also possible that persons with CKD have a lower concentration of antioxidant enzymes making them susceptible to the disease in the first place. Our results are in agreement with other studies which also found higher MDA and decreased SOD and GPx values in HD patients as compared to controls. ${ }^{[14-19]}$ All available studies in literature are unanimous in saying that MDA values increase with increasing oxidative stress. However, some studies report higher SOD values in HD patients as compared to controls. ${ }^{[20-22]}$

TAC represents the sum total of the antioxidant defence existing in the body. As CKD progresses, weakening of the antioxidant defence should correspond with lowering of TAC values. However, our results show that though TAC values in the initial stages of CKD are lower than those of controls, TAC values increase with disease progression. In fact, CKD stage 5 patients have TAC values higher than controls. This may be as a result of serum urea accumulation as the kidney function deteriorates. Previous studies too have found elevated $\mathrm{TAC}$ in later stages of CKD which were strongly correlated to higher serum creatinine, uric acid and urea levels in these patients. ${ }^{[23-24]}$

Dounousi et al reported significantly increased oxidative stress as CKD stage advanced and concluded that this plays a central role in the pathophysiological processs of uremia and its complications. ${ }^{[25]}$ According to Cachofeiro et al renal disease is associated with graded increase in oxidative stress markers even in early CKD, which could be either due to increased production of ROS or due to decrease in antioxidant defence of the body. This in turn may trigger the release of inflammatory markers like CRP and cytokines which in turn bring about deterioration of renal function. ${ }^{[26]}$ Dwivedi and Sarkar too agree that the excessive generation of ROS is one of

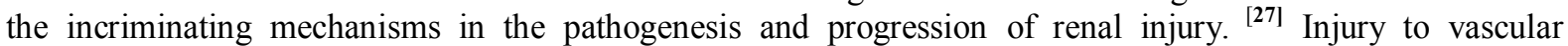
endothelium is measured by elevated levels of CRP. ${ }^{[28]}$ The degree of inflammatory response is closely related to the incidence and mortality due to cardiovascular events in CKD patients. ${ }^{[29]}$ Susla et al conclude in their study that oxidative stress and chronic inflammation are important factors in the development of cardiovascular calcification in CKD patients and this plays a very important role in the functional activity of the endothelium. ${ }^{[30]}$ The resulting atherosclerosis increases the risk of cardiovascular complications in these patients. ${ }^{[31-32]}$ The exact role of inflammation as a trigger of oxidative stress remains poorly defined and it is not yet clear as to which is the initial culprit: inflammation or oxidative stress. ${ }^{[33]}$ So it is important to identify all sources of inflammation and oxidative stress in the CKD patients. These biomarkers would prove to be excellent indicators of the underlying pathological processes and an additional aid in the diagnosis and treatment of CKD. 
Therapeutic interventions with antioxidant supplements and other free-radical scavengers may be considered as an important approach towards CKD to improve the quality of life of these patients.

\section{Conclusion}

The present study found that inflammation, increased oxidative stress and reduced antioxidant capacity are prevalent in the CKD population from the early stages. As renal function deteriorates, this situation only turns worse. The inflammation and oxidative stress may exacerbate existing disease and further deteriorate the health of the patient. It is therefore extremely important to identify oxidative stress and sources of inflammation in patients of CKD right from the time the diagnosis is made. Every effort should be made to reduce the oxidative stress in these patients. Use of antioxidant supplements should be part and parcel of their treatment plan. Longitudinal clinical trials with a larger sample size to study the beneficial effects of such supplements is definitely warranted.

\section{Acknowledgements}

The authors would like to express heartfelt thanks to all the participants of this study.

\section{REFERENCES}

[1] Jha V, Garcia-Garcia G, Iseki K, Li Z, Naicker S, Plattner B, et al, Chronic Kidney Disease: global dimension and perspectives. Lancet, 382(9888), 2013,260-72.

[2] Nimmi Singh, Rajarshi G Niyogi, Deepak Mishra, Mamta Sharma, Devika Singh, Antioxidants in Oral Health and Diseases: Future Prospects, IOSR Journal of Dental and Medical Sciences, 10(3), 2013, 36-40.

[3] Lavin-Gomez BA, Palomar-Fontanet R, Gago-Fraile M, Quintanar-Lartundo JA, Gomez-Palomo E, Gonzalez_Lamuno D, et al, Inflammation markers, chronic kidney disease, and renal replacement therapy, Adv Perit Dial., 27, 2011, 33-7.

[4] Ramamoorthy RD, Nallasamy V, Reddy R, Esther N, Maruthappan Y,A review of C-reactive protein: A diagnostic indicator in periodontal medicine, Journal of Pharmacy and BioAllied Sciences, 4(6), 2012,422-426.

[5] Massy ZA, Stenvinkle P, Drueke TB, The role of oxidative stress in CKD, Semin Dial., 22(4), 2009, $405-408$.

[6] Atamer A, Kocyigit Y, Ecder SA, Selek S, IIhan N, Ecder T, Atamer Y, Effect of oxidative stress on antioxidant enzyme activities, homocysteine and lipoproteins in chronic kidney disease, J Nephrol., 21(6), 2008, 924-30.

[7] Small DM, Coombes JS, Bennett N, Johnson DW, Gobe GC, Oxidative stress, anti-oxidant therapies and chronic kidney disease. Nephrology (Carlton), 17(4), 2012, 311-21.

[8] Kei, Satoh, Serum lipid peroxide in cerebrovascular disorders determined by a new colorimetric method, Clinica Chimica Acta, 90 , $1978,37-43$.

[9] Das K, Samanta Luna, Chainy GBN, A modified spectrophotometric assay of superoxide dismutase using nitrite formation by superoxide radicals, Indian Journal of Biochemistry and Biophysics, 37, 2000, 201-204.

[10] Paglia DE, Valentine WN,Studies on the quantitative and qualitative characterization of erythrocyte Glutathione Peroxidase, J Lab Clin Med, 70, 1967, 158-169.

[11] Benzie IF, Strain JJ, The Ferric Reducing ability of plasma (FRAP) as a measure of "antioxidant power": the FRAP assay, Anal Biochem , 239(1), 1996, 70-6.

[12] National Kidney Foundation, K/DOQI clinical practice guidelines for chronic kidney disease: evaluation, classification, and stratification, Am J Kidney Dis, 39(Suppl 2), $2002, \quad$ S1-246. Also see http://www.kidney.org/professionals/kdoqi/guidelines_ckd/p7 risk_g15.htm Part7: Stratification of risk for progression of kidney disease and development of cardiovascular disease. Viewed 10 Feb 2013.

[13] Chen SJ, Yen CH, Huang YC, Lee BJ, Hsia S, Lin PT, Relationships between inflammation, adiponectin, and oxidative stress in metabolic syndrome, PLoS One, 7(9), 2012, e45693.

[14] Richard MJ, Arnaud J, Jurkovitz C, Hachache T, Meftahi H, Laporte F, et al,Trace elements and lipid peroxidation abnormalities in patients with chronic renal failure, Nephron, 57(1), 1991, 10-5.

[15] Paul JL, Sall ND, Soni T, Poignet JL, Lindenbaum A, Man NK, Moatti N, Raichvarg D, Lipid peroxidation abnormalities in hemodialyzed patients, Nephron, 64(1), 1993,106-9.

[16] Yilmaz MI, Saglam M, Caglar K, Cakir E, Sonmez A, Ozgurtas T, et al, The determinants of Endothelial Dysfuntion in CKD: Oxidative Stress and Asymmetric Dimethylarginine, American Journal of Kidney diseases, 47(1), 2006, 42-50.

[17] Bhogade RS, Suryakar AN, Joshi NG, Patil RY, Effect of vitamin E supplementation on oxidative stress in hemodialysis patients, Indian J Clin Biochem, 23(3), 2008,-237.

[18] Nagane NS, Ganu JV, Gandhi R, Oxidative stress, serum homocysteine and serum nitric oxide in different stages of chronic renal failure, Biomedical Research, 20(1), 2009, 71-74.

[19] Guo Chih- Hung, Chen Pei- Chung, Hsu GSW, Wang CL, Zinc supplementation Alters Plasma Aluminium and Selenium Status of Patients Undergoing Dialysis: A Pilot Study, Nutrients, 5, 2013,1456- 1470.

[20] Chauhan DP, Gupta PH, Nampoothiri MRN, Singhal PC, Chugh KS, Nair CR, Determination of erythrocyte superoxide dismutase, catalase, glucose-6- phosphate dehydrogenase, reduced glutathione and malonyldialdehyde in uremia, Clin Chem Acta,123(1-2), 1982,153-159.

[21] Toborek M, Wasik T, Drozdz M, Klin M, Magner-Wrobel, Kopieczna-Grzebieniak E, Effect of hemodialysis on lipid peroxidation and antioxidant system in patients with chronic renal failure, Metabolism, 41(11), 1992,1229-32.

[22] Padalkar RK, Shinde AV, Patil SM, Lipid profile, Serum MDA, SOD in Chronic Kidney Diseases and Type 2 Diabetes Mellitus, Biomedical Research, 23(2), 2012, 207-210.

[23] Kusano C, Ferrari B, Total Antioxidant Capacity: a biomarker in biomedical and nutritional studies, Journal of Cell and Molecular Biology,7(1), 2008,1-15.

[24] Gosmanova EO, Ngoc-Anh Le, Cardiovascular Complications in CKD patients: Role of Oxidative Stress, Cardiology Research and Practice, Volume 2011, 2011, Article ID156326, 8 pages. Available at http://dx.doi.org/10.4061/2011/156326

[25] Dounousi E, Papavasiliou E, Makedou A, Ioannou K, Katopodis KP, Tselepis A, et al, Oxidative Stress is Progressively Enhanced With Advancing Stages of CKD, Am J Kid Dis, 48(5), 2006, 752-760.

[26] Cachofeiro V, Goicochea M, de Vinuesa SG, Oubina P, Lahera V, Luno J, Oxidative stress and inflammation, a link between chronic kidney disease and cardiovascular disease, Kidney Int Suppl., (111), 2008, S4- 9. 
[27] Dwivedi J, Sarkar PD, Lipoproteins (A), homocystiene, Lipid Profile with oxidative stress in Nephrotic Syndrome and Cardiovascular Nephropathy, International Journal of Pharma and Bio Sciences, 1(4), 2010, B340-350.

[28] Cleland SJ, Sattar N, Petrie JR, Forouhi NG, Elliott HL, Connell JM. Endothelial dysfunction as a possible link between C-reactive protein levels and cardiovascular disease. Clin Sci (Lond), 98(5), 2000,531-5

[29] Menon V, Greene T, Wang X, Pereira AA, Marcovina SM, Beck GJ, et al, C-Reactive Protein and albumin as predictors of allcause and cardiovascular mortality in chronic kidney disease. Kidney Int, 68, 2005, 766-772.

[30] Susla Oleksandr, Oxidative stress and systemic inflammation as important components of cardiac valve calcification in predialysis patients, Nephrol. Dial. Transplan., 27(suppl 2), 2012, ii405-ii413.

[31] Cottone S, Lorito MC, Riccobene R, Nardi C, Mul G, Buscemi S, et al, Oxidative Stress, inflammation and cardiovascular disease in Chronic Renal Failure, J Nephrol, 21(2), 2008,175-179.

[32] Zalba G, Fortuno Ana, Diez J, Oxidative stress and atherosclerosis in early CKD, Nephrol. Dial. Transplant., 21(10), 2006,26862690.

[33] Stenvinkel P, Carrerp KK, Axelsson J, Lindholm B, Heimburger O, Massy Z, Emerging Biomarkers for Evaluating Cardiovascular Risk in the Chronic Kidney Disease Patient: How do New Pieces Fit into the Uremic Puzzle? Clinical Journal of the Am Soc of Nephrol, 3(2), 2008, 505-521. 